Objectives In Australian MSBase clinics, we describe baseline characteristics of relapsing-remitting MS (RRMS) patients treated with OCR, treatment pathways and early clinical outcomes.

Methods Secondary analysis using MSBase Registry data for RRMS patients with OCR initiation within 3 months of MSBase recorded visit. Descriptive statistics included demographics, disease course/duration, prior disease modifying therapies (DMT) and EDSS. Relapse data was described in patients with $\geq 6$ months follow-up.

Results As of 4 June 2020, MSBase included 624 eligible Australian RRMS patients newly treated with OCR. Median age at OCR initiation was 42.5 years. OCR was first line therapy in $18.9 \%$ of patients. Most frequent DMT's in the 12 months prior to OCR were natalizumab (32.1\%) and fingolimod (24.8\%). Of 434 RRMS patients with $\geq 6$ months follow-up, 392 remained relapse free $(90.3 \%$; 95\% CI 81.6, 99.7) over a mean OCR exposure of 1.35 years. In this group, the annualized relapse rate (ARR) was 0.10 (95\% CI $0.08-0.13$ ), compared to an ARR of 0.83 in the 24 months pre-OCR start. Treatment discontinuation was recorded for 20 of these 434 patients In the overall RRMS cohort, treatment persistence at 12 and 24 months was 94.3\% (95\%CI: 90.9\%96.1\%\%) and $88.7 \%$ (95\%CI 77.2\%-94.0\%), respectively.

Conclusion Almost 20\% of RRMS patients treated with OCR in Australian MSBase centres received OCR as a first line treatment. During OCR treatment, relapses and OCR discontinuations were rare.

\section{WORSENING LONGITUDINAL REACTION TIME TRAJECTORIES USING THE MSREACTOR COMPUTERISED BATTERY PREDICTS CONFIRMED EDSS PROGRESSION}

${ }^{1}$ Daniel Merlo, ${ }^{1} \mathrm{Jim}$ Stankovich, ${ }^{2}$ Claire Bai, ${ }^{2}$ Tomas Kalincik, ${ }^{1}$ Melissa Gresle, ${ }^{3}$ Jeannette Lechner-Scott, ${ }^{4}$ Trevor Kilpatrick, ${ }^{5}$ Michael Barnett, ${ }^{6}$ Bruce Taylor, ${ }^{4}$ David Darby, ${ }^{1}$ Helmut Butzkueven, ${ }^{1}$ Anneke van der Walt. ${ }^{1}$ MSNI, Central Clinical School, Monash University, Melbourne, VIC, Australia; ${ }^{2}$ CORe, Department of Medicine at RMH, University of Melbourne, Melbourne, VIC, Australia; ${ }^{3}$ Department of Neurology, John Hunter Hospital, Newcastle, NSW, Australia; ${ }^{4}$ Florey Institute of Neuroscience and Mental Health, Melbourne, VIC, Australia; ${ }^{5}$ Brain and Mind Centre, Sydney, NSW, Australia; ${ }^{6}$ Department of Neurology, Royal Hobart Hospital, Hobart, TAS, Australia

\subsection{6/bmjno-2021-ANZAN.11}

Objectives To identify and validate longitudinal reaction time trajectories in relapsing remitting multiple sclerosis using a computerised cognitive battery and latent class mixed modelling, and to assess the association between reaction time trajectories and disability progression.

Methods Participants serially completed web-based computerised reaction time tasks measuring psychomotor speed, visual attention and working memory. Testing sessions were completed 6-monthly with the option of additional home based testing. Participants who completed at least three testing sessions over a minimum of 180 days were included in the analysis. Longitudinal reaction times were modelled using Latent Class Mixed Models to group individuals sharing similar latent characteristics. Models were tested for consistency using a cross-validation approach. Inter-class differences in the probability of reaction time worsening and the probability of 6month confirmed disability progression were assessed using survival analysis.

Results A total of 460 relapsing remitting multiple sclerosis patients were included. For each task of the MSReactor computerised cognitive battery, the optimal model comprised of 3 latent classes. All tasks could identify a group with high probability of reaction time slowing. The visual attention and working memory tasks could identify a group of participants who were 3.7 and 2.6 times more likely to experience a 6-month confirmed disability progression, respectively. Participants could be classified into predicted cognitive trajectories after just 5 tests with between $64 \%$ and 89\% accuracy.

Conclusion Latent class modelling of longitudinal cognitive data collected by the MSReactor battery identified a group of patients with worsening reaction times and increased risk of disability progression.

\section{VOLUMETRIC AND CONNECTIVITY PROFILE OF REGIONAL THALAMIC ABNORMALITY IN AMYOTROPHIC LATERAL SCLEROSIS}

${ }^{1}$ Sicong Tu, ${ }^{2}$ Marion Sourty, ${ }^{1}$ Fernando Calamante, ${ }^{3}$ Manoj Saranathan, ${ }^{4}$ Ricarda Menke, ${ }^{4}$ Kevin Talbot, ${ }^{1}$ Matthew Kiernan, ${ }^{4}$ Martin Turner. ${ }^{1}$ Brain and Mind Centre, University of Sydney, Sydney, NSW, Australia; ${ }^{2}$ Université de Strasbourg, Strasbourg, France; ${ }^{3}$ University of Arizona, Tucson, Arizona, USA; ${ }^{4}$ Nuffield Department of Clinical Neurosciences, University of Oxford, Oxford, UK

\subsection{6/bmjno-2021-ANZAN.12}

Objectives Neurodegeneration in ALS follows a diffuse pattern of cortical involvement. ${ }^{1}$ We have previously highlighted that thalamic abnormality is a robust disease signature in $\mathrm{ALS},{ }^{2}$ but the integrity of thalamic nuclei and their clinical association remains unclear. We employed a novel segmentation technique for thalamic nuclei and track-weighted functional connectivity (TW-sFC) to characterize volumetric and connectivity profiles of regional thalamic abnormality.

Methods Forty ALS patients and 27 age-and-education matched controls were recruited. All patients underwent comprehensive clinical examination and 3T MRI scan (T1; DWI; rs-fMRI). Thalamic nuclei were robustly segmented from T1 images using the THOMAS pipeline. ${ }^{3}$ Whole-brain white matter fibre tracking was performed using MRtrix and combined with resting-state fMRI to generate combined structural and functional connectivity maps (TW-sFC). ${ }^{4}$

Results Reduced thalamus volume was observed bilaterally in ALS compared to control ( $\mathrm{p}$ values < 0.036). Bilateral volumetric reduction was consistently observed across all regions except for the anterior thalamus in ALS ( $p$ values $<0.05$ ). Significant increased TW-sFC was observed in ALS in the right anterior thalamus $(\mathrm{p}=0.03)$ and right anterior ventral nuclei ( $\mathrm{p}<0.01$ ). TW-sFC of the mediodorsal nuclei correlated with disease duration $(\mathrm{p}<0.02)$ and disease progression rate $(\mathrm{p}<0.03)$.

Conclusions Regional thalamic abnormalities are present in ALS and hold a significant association with clinical features. Variability in thalamic connectivity demonstrated significant clinical associations with disease duration, progression rate, and upper motor dysfunction. The findings reinforce that diffusion and functional MR imaging modalities are promising markers of disease burden in ALS.

\section{REFERENCES}

1. Brettschneider J, Del Tredici K, Toledo J, et al. Stages of pTDP-43 pathology in amyotrophic lateral sclerosis. Ann Neurol 2013;74:20-38.

2. Tu S, Menke R, Talbot K, Kiernan M, Turner M. Regional thalamic MRI as a marker of widespread cortical pathology and progressive frontotemporal involvement in amyotrophic lateral sclerosis. JNNP 2018;89:1250-1258. 
3. Su J, Thomas F, Kaso W, et al. Thalamus optimized multi atlas segmentation (THOMAS): fast, fully automated segmentation of thalamic nuclei from structural MRI. Neuroimage 2019;194:272-282.

4. Calamante F, Smith R, Liang X, Zalesky A, Connelly A. Track-weighted dynamic functional connectivity (TW-dFC): a new method to study time-resolved functional connectivity. Brain Struct Funct 2017;222:3761-3774.

\section{PROGRESSION OF CLINICAL FEATURES IN LEWY BODY DEMENTIA CAN BE DETECTED OVER SIX MONTHS}

\begin{abstract}
1,2Elie Matar, ${ }^{3}$ Simon R White, ${ }^{4}$ John P Taylor, ${ }^{5}$ Alan Thomas, ${ }^{4}$ lan G McKeith, ${ }^{6} J o s e p h$ PM Kane, ${ }^{2}$ AJ Surendranathan, ${ }^{1}$ Glenda M Halliday, ${ }^{1}$ Simon JG Lewis, ${ }^{2} J o h n$ T O'Brien. ${ }^{1}$ Brain and Mind Centre, University of Sydney, Camperdown, NSW, Australia; ${ }^{2}$ Department of Psychiatry, University of Cambridge, Cambridge, Cambridgeshire, UK; ${ }^{3}$ MRC Biostatistics Unit, University of Cambridge, Cambridge, UK; ${ }^{4}$ Newcastle Translational and Clinical Research Institute, Newcastle University, Newcastle upon Tyne, UK; ${ }^{5}$ Campus for Ageing and Vitality, Newcastle University, Newcastle, UK; ${ }^{6}$ Centre for Public Health, Queen's University Belfast, Belfast, UK
\end{abstract}

10.1136/bmjno-2021-ANZAN.13

Objective This study aimed to quantify the trajectory and magnitude of change of the key clinical features and corresponding symptom domains of Dementia with Lewy bodies (DLB) and Parkinson's disease dementia (PDD) including global cognition, parkinsonism, recurrent visual hallucinations, cognitive fluctuations and sleep disturbance.

Methods 116 patients with Lewy body dementia (DLB $=72$, $\mathrm{PDD}=44$ ) underwent assessment at baseline, 3 and 6 months as part of a prospective multi-centre randomized control trial. Linear mixed models were constructed for core outcome measures using the Mini-Mental State Examination (MMSE), motor section of the Unified Parkinson's disease rating scale (UPDRS-III), Dementia Cognitive Fluctuations Scale (DCFS) and the Neuropsychiatric Inventory (NPI).

Results Within the timeframe of our study (6 months) we were able to identify a significant cognitive decline of 1.3 points on the MMSE $(\mathrm{P}=0.002)$ and significant worsening of motor parkinsonism with an increase in UPDRS-III score of 3.2 points $(\mathrm{P}=0.018)$. Fluctuation severity also increased using the DCFS with a 6 month change in score of 1.3 points $(\mathrm{P}=0.001)$. Uniquely, a signal for increased severity of sleep symptoms of 1.2 points (NPI-sleep) was also detectable $(\mathrm{P}=0.04)$. Significant changes in neuropsychiatric symptoms were not detected. There was no difference in rates of change of scores between DLB and PDD.

Conclusion Clinically significant rates of change in core clinical features can be detected and quantified in Lewy body dementia over a relatively short period (6 months) using common clinical instruments, and thus may be useful as clinical endpoints for therapeutic trials of disease modifying and symptomatic agents.

\section{VISUALISING THE TOPOGRAPHIC PATTERN OF TAU DEPOSITION IN PATIENTS WITH PROGRESSIVE SUPRANUCLEAR PALSY USING PI2620-PET}

1,2Lucy Vivash, ${ }^{2}$ Huiliang Tang, ${ }^{1,2}$ Kelly Bertram, ${ }^{1,2}$ Terence J O'Brien. 'Monash University, Melbourne, VIC, Australia; ${ }^{2}$ Alfred Hospital, Melbourne, VIC, Australia

\subsection{6/bmino-2021-ANZAN.14}

Objectives The accumulation of tau in the basal ganglia and later cerebellum and frontal cortex is a key pathogenic mechanism in Progressive Supranuclear Palsy (PSP). The ability to detect tau in the living brain has the potential to revolutionise the diagnosis of PSP and other tauopathies, and to monitor the effectiveness of therapeutic interventions targeting tau-based mechanisms. This study investigates the use of a new tau-specific PET radiotracer, PI-2620, as a tool for visualising tau in the living brain.

Methods Ten patients with PSP (age 62-75 years, 6 male) underwent a 60 minute dynamic PET scan with PI2620 $(185 \mathrm{MBq})$. The dynamic PET data was processed in to a single parametric image of binding potential (BP) using the simplified reference tissue model and the corpus callosum as the reference region.

Results Visual inspection of the images showed clear uptake in the basal ganglia nuclei. BP was highest in pallidum (1.99), putamen (1.67), thalamus (1.63), substantia nigra (1.62), cerebellum (1.6) and caudate (1.55), compared to 1.2-1.5 across the cortical regions. Higher $\mathrm{BP}$ in the basal ganglia nuclei were associated with higher scores on the PSPRS (putamen, $\mathrm{r}=0.77, \mathrm{p}=0.01)$.

Conclusion PI2620-PET shows promising potential as a technique for specifically imaging and quantitating the topographic pattern of tau distribution in patients with PSP. Further studies are needed to evaluate its use as a diagnostic and treatment monitoring tool for PSP and other tauopathies.

\section{GUT MICROBIOTA AND NUTRITIONAL PROFILES OF PARKINSON'S DISEASE PATIENTS}

${ }^{1,2}$ Michal Lubomski, ${ }^{3}$ Xiangnan $\mathrm{Xu},{ }^{4}$ Andrew J Holmes, ${ }^{3}$ Samuel Mueller, ${ }^{3}$ Jean Yang, ${ }^{1,2}$ Carolyn M Sue, ${ }^{2}$ Ryan L Davis. 'Neurology Department, Royal North Shore Hospital, St Leonards, NSW, Australia; ${ }^{2}$ Department of Neurogenetics, Kolling Institute, University of Sydney and Royal North Shore Hospital, Sydney, NSW, Australia; ${ }^{3}$ School of Mathematics and Statistics. Sydney Precision Bioinformatics, University of Sydney, Camperdown, NSW Australia; ${ }^{4}$ School of Life and Environmental Sciences, The Charles Perkins Centre, University of Sydney, Sydney, NSW, Australia

\subsection{6/bmjno-2021-ANZAN.15}

Objectives Imbalances in the composition of the gut microbiome (GM) from Parkinson's disease (PD) patients have been reported previously. Collectively, the limited literature indicates a reduction in short-chain fatty-acid-producing bacteria that negatively influence colonic permeability and inflammation. We investigated GM composition in association with various clinical features and nutritional data in a large cross-sectional Australian PD cohort, to determine whether short-chain fattyacid-producing bacteria representation in the GM was altered in association with clinical or nutritional differences between PD patients and controls.

Methods Clinical outcome measures derived from PD-validated questionnaires and stool samples were collected from 103 PD patients and 81 spousal healthy controls (HCs). GM composition, determined from $16 \mathrm{~S}$ amplicon sequencing of the V3-V4 region of stool bacterial DNA, was compared between groups and with clinical outcome measures.

Results We identified significant compositional differences in the GM profiles of PD patients compared to HCs, across order, family and genus taxonomic levels. Multiple taxa were associated with a variety of clinical PD characteristics. Predictive models using GM profiles were developed to identify PD and were improved by incorporating nutritional data. 